\title{
Leukocytoclastic vasculitis as a previously unreported paraneoplastic manifestation of acute lymphoblastic leukemia in adults
}

\author{
Rashad Ismayilov $^{1}$ (D) Tahmaz Haziyev $^{2}$ (D) Deniz Ates Ozdemir $^{3}$ (D) Arzu Saglam $^{3}$ (D) Yahya Buyukasik $^{2}$ (D)
}

Received: 4 September 2020 / Accepted: 22 September 2020 / Published online: 29 September 2020

(C) Springer-Verlag GmbH Germany, part of Springer Nature 2020

\begin{abstract}
Background Although leukocytoclastic vasculitis (LCV) is often idiopathic or secondary to benign diseases, it sometimes accompanies malignancies. Besides being more common in hematological cancers compared to solid tumors, the association of acute lymphoblastic leukemia (ALL) with LCV is extremely rare.

Case report In this study, we present a 22-year-old woman presenting with palpable petechiae, purpura, and ecchymoses on her limbs. Bone marrow and skin biopsy of the patient with bicytopenia revealed precursor B cell ALL and LCV, respectively.

Conclusion This case is the first adult ALL case with paraneoplastic LCV. In this study, it is emphasized that hematological malignancies should be considered in patients presenting with cutaneous vasculitis, especially in the presence of cytopenia.
\end{abstract}

Keywords ALL $\cdot$ Leukocytoclastic vasculitis $\cdot$ Cutaneous vasculitis $\cdot$ Paraneoplastic syndrome

\section{Introduction}

Leukocytoclastic vasculitis (LCV) is a histopathological definition and is characterized by inflammation in small vessels of the skin and the breakdown of neutrophils [1]. Cutaneous vasculitis can present in different ways: (a) as cutaneous component of systemic vasculitis, (b) as skin-limited or skin-

Rashad Ismayilov

ismayilov_r@hotmail.com

Tahmaz Haziyev

doctor.heziyev@gmail.com

Deniz Ates Ozdemir

denizatesozdemir@gmail.com

Arzu Saglam

eminearzusaglam@gmail.com

Yahya Buyukasik

ybuyukas@hacettepe.edu.tr

1 Department of Internal Medicine, Hacettepe University Faculty of Medicine, Ankara, Turkey

2 Department of Hematology, Hacettepe University Faculty of Medicine, Ankara, Turkey

3 Department of Pathology, Hacettepe University Faculty of Medicine, Ankara, Turkey dominant variant of systemic vasculitis, and (c) as single organ vasculitis of the skin [2]. Although single-organ vasculitis of the skin is more often idiopathic, it can also develop secondary to many causes such as drugs, infections, connective tissue diseases, and malignancies. Paraneoplastic cutaneous vasculitis occurs at a rate of $3.8 \%$ and is more common in hematological malignancies than in solid tumors [3]. To our knowledge, the patient we present is the first adult case of ALL with paraneoplastic LCV.

\section{Case presentation}

A 22-year-old woman presented with acute complaints of erythematous rashes on the extremities. The patient did not have weight loss, night sweats, myalgia, arthralgia, hematuria, abdominal pain, diarrhea, melena, chest pain, cough, dyspnea, hemoptysis, sputum, nor dysuria. She had no previously known illness, allergies, history of any drug use, a family history of malignancy nor rheumatological disease, and did not smoke. On physical examination, non-blanching palpable purpura, petechiae, and ecchymoses were detected on the forearms and legs (Fig. 1a). There was no lymphadenopathy and hepatosplenomegaly and other system examinations were normal. In laboratory investigation, hemoglobin concentration was $8 \mathrm{~g} / \mathrm{dL}(11.7-15.5)$, leukocyte count was $1.1 \times 10^{3} / \mu \mathrm{L}$ 
Fig. 1 a Extensive nonblanching palpable purpura, petechiae, and ecchymosis on the legs. b Three weeks after starting ALL treatment

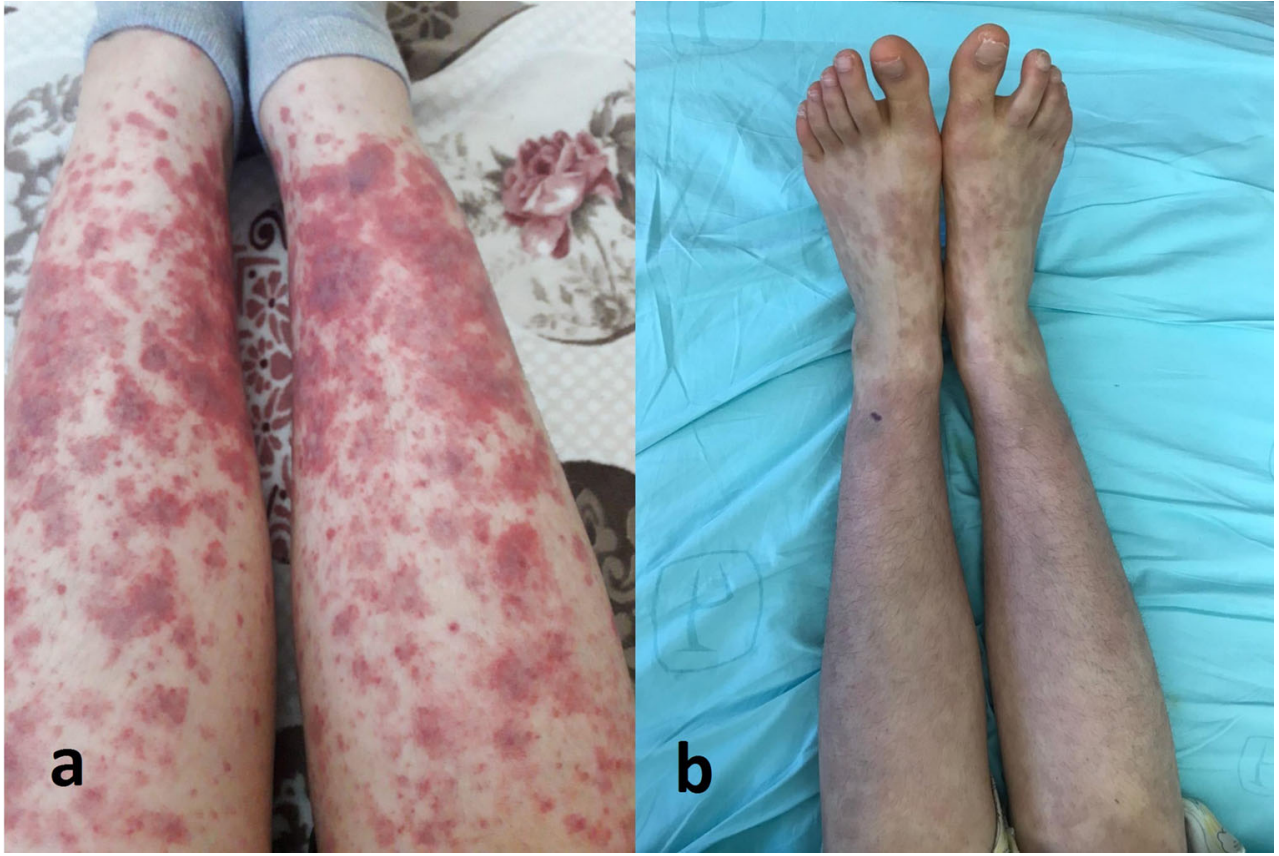

$\left(4.1-11.2 \times 10^{3}\right)$ with $51.9 \%$ lymphocytes, $2.2 \%$ monocytes, $44.9 \%$ neutrophils, and $0.3 \%$ eosinophils, and platelet count was $160 \times 10^{3} / \mu \mathrm{L}\left(159-388 \times 10^{3}\right)$. There was no abnormality in liver and kidney function tests and complete urine analysis. Serology for hepatitis B surface antigen (HBsAg), hepatitis C virus (HCV), and human immunodeficiency virus (HIV) were negative. Rheumatoid factor (RF) was $34.2 \mathrm{IU} / \mathrm{mL}(0-20)$, anti-nuclear antibody (ANA) test was positive with 1:160 titer speckled pattern, $\mathrm{C} 3$ and $\mathrm{C} 4$ were normal, cyclic citrullinated peptide (CCP), anti-dsDNA, anti-Sm, anti-Ro, anti-La, antiJo-1, anti-Scl-70, and antineutrophil cytoplasmic antibodies (ANCA) were negative.

The patient underwent bone marrow aspiration biopsy and a punch biopsy of the skin from ecchymotic lesions on the leg. Skin biopsy revealed fibrin deposition in superficial small vessels in the dermis, vascular wall destruction, erythrocyte extravasation, and leukocytoclasia (Fig. 2). In the direct immunofluorescence examination, perivascular $\mathrm{C} 3$ and $\operatorname{IgM}$ were positive and $\operatorname{IgA}$ and $\operatorname{IgG}$ were negative. The diagnosis of LCV was made. Bone marrow biopsy revealed hypercellular marrow tissue with diffuse blastic infiltration (Fig. 3). In immunohistochemical examination, blastic cells were positive with $\mathrm{CD} 34$, terminal deoxynucleotide transferase (TdT), and CD19 and focally positive with CD10. The findings were consistent with precursor B cell acute lymphoblastic leukemia (ALL). In the flow cytometry, CD10 (64\%), CD19 (97\%), cCD79a (50\%), and TdT (45\%) were positive. Bone marrow cytogenetics revealed a normal $46, X X$ female karyotype.

Brain, neck, thorax, and abdominal computed tomography (CT) examinations revealed no pathological findings. There was no central nervous system (CNS) involvement on cerebral spinal fluid (CSF) examination.

Induction chemotherapy with the pediatric ALL regimen BFM-95 IA was instituted with vincristine, daunorubicine, prednisone, and asparaginase as well as intrathecal methotrexate [4]. Significant improvement was observed in skin lesions within 3 weeks after first induction regimen (Fig. 1b). She
Fig. 2 a The walls of the papillary dermal capillaries are destructed (arrows). Fibrinoid change and leukocytoclastic karyorrhexis are visible $(\mathrm{H} \& \mathrm{E}, \times$ 200). b Fibrinoid change, leukocytoclasis, and minimal erythrocyte extravasation at high power field $(\mathrm{H} \& \mathrm{E}, \times 400)$

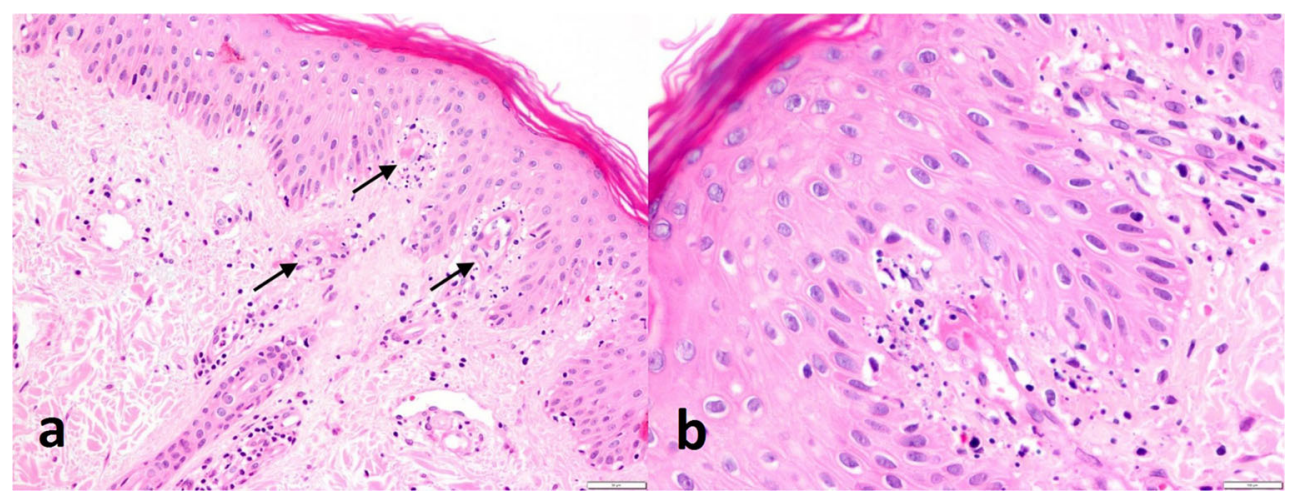




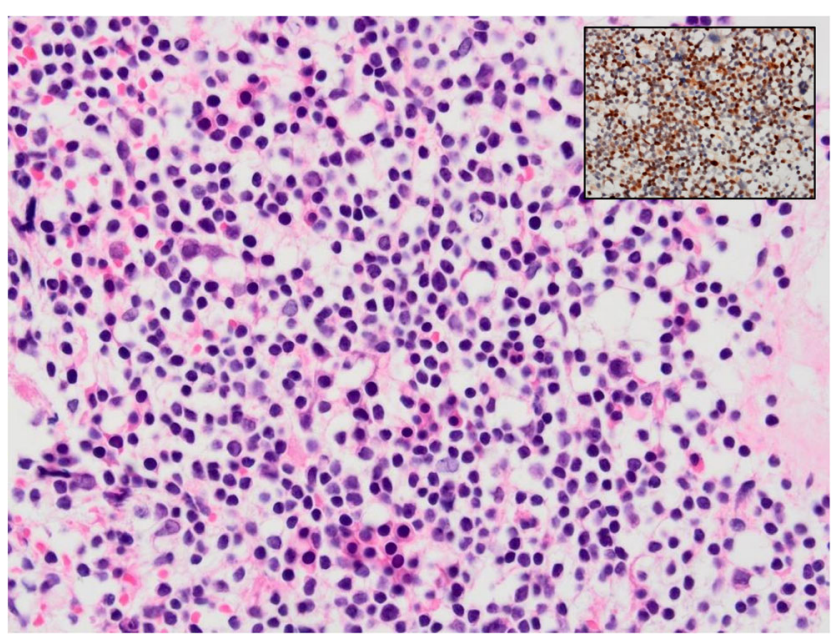

Fig. 3 Bone marrow biopsy. A diffuse infiltrate composed of monotonous mononucleated cells, some with discernible blastic nuclei can be seen with the H\&E stain. Inset show immunohistochemical Tdt positivity

achieved complete remission with minimal residual disease (MRD) negativity, and we started second-phase induction protocol BFM-95 IB consisting of cyclophosphamide, cytarabine, mercaptopurine, and intrathecal methotrexate.

\section{Discussion}

In 1986, McLean described two criteria for the identification of paraneoplastic vasculitis: first, the simultaneous occurrence of vasculitis and neoplasia; and second, their parallel course [5]. Cutaneous LCV is the most common paraneoplastic vasculitis with a rate of $43-45 \%$, and it is more common in hematological malignancies than in solid tumors $[6,7]$. There are cases reported with many hematological malignancies, such as acute myeloid leukemia (AML), chronic lymphocytic leukemia (CLL), hairy cell leukemia, lymphomas, multiple myeloma, and myeloproliferative diseases [8-11]. But paraneoplastic cutaneous vasculitis in ALL is extremely rare and has been previously reported only in an 11-year-old girl [12]. To the best of our knowledge, our patient is the first adult ALL case with paraneoplastic LCV.

The exact mechanism of the development of paraneoplastic vasculitis is not yet known. In 1986, Longley et al. initially suggested that malignant neoplasms can produce antigens, causing paraneoplastic vasculitis [13]. Besides this, several possible mechanisms have been suggested such as impaired clearance of normally produced immune complexes and production of antibodies directed toward endothelial antigens [3]. Infections are common in patients with cancer, and it can be a confusing factor in understanding the relationship between vasculitis and malignancy. In one study, confounding factors such as infection, drug reactions, and cryoglobulin deposition were detected in $39 \%$ of cases [11].
In most cases, cancer treatment also improves paraneoplastic vasculitis. When curative treatment cannot be applied, glucocorticoids or other immunosuppressive agents may be needed for vasculitis treatment [3]. In our patient, improvement of cutaneous lesions was observed within 3 weeks after BFM-95 chemotherapy was commenced.

Paraneoplastic vasculitis is diagnosed simultaneously with malignancies in $38 \%$ of cases [3, 7]. Histopathologically, LCV constitutes the vast majority of paraneoplastic cutaneous vasculitis, and this is important especially in the initial diagnosis. Our case highlights that in cases with cutaneous LCV, clinicians should be aware that this may accompany hematological malignancies, especially in the presence of cytopenias.

Data Availability The data that support the findings of this study are available from the corresponding author, upon reasonable request.

\section{Compliance with ethical standards}

Conflict of interest The authors declare that they have no conflict of interest.

\section{Ethics approval Not applicable}

Consent to participate Written informed consent for participation of their details was obtained from the patient.

Consent for publication Written informed consent for publication of their details was obtained from the patient.

Code availability Not applicable

\section{References}

1. Carlson JA, Ng BT, Chen K (2005) Cutaneous vasculitis update: diagnostic criteria, classification, epidemiology, etiology, pathogenesis, evaluation and prognosis. Am J Dermatopathol. 27(6): 504-528

2. Sunderkötter CH, Zelger B, Chen KR, Requena L, Piette W, Carlson JA et al (2018) Nomenclature of cutaneous vasculitis: dermatologic addendum to the 2012 Revised International Chapel Hill Consensus Conference Nomenclature of Vasculitides. Arthritis Rheumatol. 70(2):171-184

3. Loricera J, Calvo-Río V, Ortiz-Sanjuán F, González-López MA, Fernández-Llaca H, Rueda-Gotor J et al (2013) The spectrum of paraneoplastic cutaneous vasculitis in a defined population: incidence and clinical features. Med (United States). 92(6):331-343

4. Avramis VI, Sencer S, Periclou AP, Sather H, Bostrom BC, Cohen LJ et al (2002) A randomized comparison of native Escherichia coli asparaginase and polyethylene glycol conjugated asparaginase for treatment of children with newly diagnosed standard-risk acute lymphoblastic leukemia: a Children's Cancer Group study. Blood. 99(6):1986-1994

5. McLean DI (1986) Cutaneous paraneoplastic syndromes. Arch Dermatol. 122:765-767

6. Kurzrock R, Cohen PR (1993) Vasculitis and cancer. Clin Dermatol. 11(1):175-187 
7. Fain $\mathrm{O}$, Hamidou M, Cacoub $\mathrm{P}$, Godeau B, Wechsler B, Pariès J et al (2007) Vasculitides associated with malignancies: analysis of sixty patients. Arthritis Care Res. 57(8):1473-1480

8. Jayachandran NV, Thomas J, Chandrasekhara PKS, Kanchinadham S, Kadel JK, Narsimulu G (2009) Cutaneous vasculitis as a presenting manifestation of acute myeloid leukemia. Int J Rheum Dis. 12(1):70-73

9. Lulla P, Bandeali S, Baker K (2011) Fatal paraneoplastic systemic leukocytoclastic vasculitis as a presenting feature of chronic lymphocytic leukemia. Clin Lymphoma. Myeloma Leuk 11(SUPPL.1):S14-S16

10. Moyers JT, Liu LW, Ossowski S, Goddard L, Kamal MO, Cao H (2019) A rash in a hairy situation: leukocytoclastic vasculitis at presentation of hairy cell leukemia. Am J Hematol. 94(12):14331434
11. Bachmeyer C, Wetterwald E, Aractingi S (2005) Cutaneous vasculitis in the course of hematologic malignancies. Dermatology. 210(1):8-14

12. Jaing TH, Hsueh C, Chiu CH, Shih IH, Chan CK, Hung IJ (2002) Cutaneous lymphocytic vasculitis as the presenting feature of acute lymphoblastic leukemia. J Pediatr Hematol Oncol. 24(7):555-557

13. Longley S, Caldwell JR, Panush RS (1986) Paraneoplastic vasculitis. Unique syndrome of cutaneous angiitis and arthritis associated with myeloproliferative disorders. Am J Med. 80(6):1027-1030

Publisher's note Springer Nature remains neutral with regard to jurisdictional claims in published maps and institutional affiliations. 